\title{
Multi-Objective Spider Monkey Optimization for Energy Efficient clustering and Routing in wireless sensor networks
}

\section{Duraimurugan Samiayya}

St. Joseph's College of Engineering

Avudaiammal Ramalingam ( $\nabla$ dravudaiece@gmail.com )

St. Joseph's College of Engg

\section{Research Article}

Keywords: Wireless sensor network (WSN), load balancing, clustering, routing and spider monkey optimization (SMO)

Posted Date: June 4th, 2021

DOI: https://doi.org/10.21203/rs.3.rs-412238/v1

License: (c) (i) This work is licensed under a Creative Commons Attribution 4.0 International License.

Read Full License 


\title{
Multi-Objective Spider Monkey Optimization for Energy Efficient clustering and Routing in wireless sensor networks
}

\author{
Duraimurugan $S^{1}$,Avudaiammal $\mathbf{R}^{2}$ \\ ${ }^{1}$ Department of Information Technology, St. Joseph's College of Engineering, Chennai-600 \\ 119, Tamil Nadu, India; \\ duraimurugans@stjosephs.ac.in \\ ${ }^{2}$ Department of Electronics and Communication, St. Joseph's College of Engineering, Chennai- \\ 600 119, Tamil Nadu, India; \\ avudaiammalr@stjosephs.ac.in
}

\begin{abstract}
In wireless sensor network (WSN), the gateways far away from the base station (BS) uses the gateways nearer to the BS to forward the data. It causes heavy traffic to the gateways in proximity with the BS. They need to manage this heavy traffic load but it leads to additional energy consumption and reduction in network lifetime. In order to overcome these issues, loads around the gateways need to be balanced. In this paper, multi objective based spider monkey optimization (MOSMO) has been presented to balance the load and to improve the network lifetime through energy efficient routing and clustering. The objective functions such as routing fitness and clustering fitness have been considered for optimal routing and clustering. The routing fitness function is found by incorporating both the minimum distance traversed by the gateways and minimum number of the gateway hops. The clustering fitness function is the minimum fitness function of gateways. The fitness function of each gateway is computed based on both the mean load of gateways as well as the distance between gateways and BS. The performance of the proposed MOSMO based routing and clustering scheme is compared with the existing Particle Swarm Optimization (PSO) and Grey Wolf Optimization (GWO) based routing and clustering scheme. The QoS features such as delay, energy consumption, delivery ratio, throughput and network lifetime with various node density are analyzed. The proposed work is simulated using MATLAB. The results show that, the reduction in delay and energy consumption is about $18 \%$ and $17 \%$ respectively whereas improvement in delivery ratio, throughput and network life time is about $15 \%, 24 \%$ and $19 \%$ respectively when compared to the existing PSO and GWO methods.
\end{abstract}

Keywords: Wireless sensor network (WSN), load balancing, clustering, routing and spider monkey optimization (SMO). 


\section{Introduction}

Wireless sensor networks (WSNs) are evolving to provide affordable and adaptable solutions in environmental monitoring, habitat monitoring, home automation and smart grids[1] [2]. More number of sensor nodes are required in medical, military surveillance, weather forecasting, industrial and commercial applications Kaushik et al to guarantee acceptable coverage and to reduce node failure [22]. Nevertheless, a closely populated WSN cause interference between nodes, several transmission routes, consumption of more energy by each node during their communication with distant nodes. Moreover, a routing path needs to be updated even when the there is a slight topological change. The sensor nodes [3] collect data from the environment and the cluster head $(\mathrm{CH})$ present in the sensor nodes communicate with the Base Station (BS) either directly or through the gateway [5] [6]. In direct mode, each node has a communication channel between the sensor and the BS [4]. The gateway prevents rapid loss of electricity through $\mathrm{CH}$ [7][8], Remote BS Ports sends data to the nearest gateway of BS. As a result, gateways carry more traffic loads and the data communication consumes more percentage of energy [16], it exhausts more of their energy quickly [9]. It may cause a power gap around the BS station and may lead to the network disconnection and thereby BS may be disconnected from the remote gateway. Therefore, network load balancing management [10] is required.

The sensor nodes have very less power as they are driven by a non-rechargeable battery and hance their power need to be exploited appropriately to improve network lifetime. The network lifetime can be improved by increasing the life time of each and every sensor node. Even the failure of a single node may reduce the network performance abruptly. Thus, the lifetime of the network depends on three factors such as, lifetime of the nodes, their coverage, and their connectivity [25]. Though, current developments in the design of electronic circuits help in producing cost effective, energy efficient sensors with lighter weight, the energy has to be effectively used for avoiding node failures and in turn network failure. Maintaining the energy of sensor nodes in WSNs for achieving an extensive network time becomes a great challenge for the researchers. The energy-concerned issues in WSNs have triggered the researchers in developing several clustering and routing approaches for enhancing the network lifetime. Clustering in WSNs refers splitting the network into a number of clusters, each with smaller number of member nodes with one $\mathrm{CH}$ [21]. Clustering helps in reducing long range 
communications, total energy consumption, channel contention and packet collision. It also helps in achieving higher throughput under heavy load [25]. The factors influence the clustering performance are the creation of optimal number of clusters and the procedure of selecting $\mathrm{CH}$ [26]. Since, the clustering in WSNs is a NP-hard problem,_various metaheuristic procedures are exploited to optimize clustering [24]. Nowadays, researchers focus towards algorithms based on Particle Swarm Optimization (PSO), Grey Wolf Optimization (GWO) an Spider Monkey Optimization (SMO) to perform clustering and routing. PSO is a smart heuristic algorithm in which the solution of the problem is arrived by sensing the knowledge of swarm, the location of the particles and the fitness function. During each iteration the location of the particles in the searching space is upgraded through the selection of local and global optimal solutions. Eventually, the particles arrive the optimal solution after a number of iterations. GWO is a swarm intelligence algorithm which mimics the hierarchical structure of wolf packs of $5-12$ wolves. Each wolf fits in only one level, in the hierarchy where alpha positions at the top and acts as a leader in taking all the vital decisions. The next level packs are the beta wolves which follows the alpha and can substitute the death alpha wolves. The last level wolves are the Omega wolves who are the caretakers of the pack but obey the commands of all the members of the pack. The other type of wolves are the deltas. This hierarchical concept of GWO is exploited in choosing CHs [25]. Spider Monkey Optimization (SMO) is another optimization algorithm stirred by hunting behavior of spider monkeys that follows Fission-Fusion social structure [23]. Self-organization and labor partitioning are the two essential notions of swarm intelligence. The update functions are computed by finding Euclidean distances between potential solutions.

In this paper, multi objective based spider monkey optimization (MOSMO) has been proposed to balance the load and to improve the network lifetime through energy efficient routing and clustering. The objectives of the paper are:

- To present a spider monkey optimization (SMO) for load balancing.

- To employ routing fitness, clustering fitness for finding the best route from the gateway to the BS.

- To implement the proposed MOSMO in the MATLAB platform.

- To analyze performance metrics such as delay, energy consumption, delivery ratio, throughput and network lifetime in various node density with the existing Particle Swarm 
Optimization (PSO) and Grey Wolf Optimization (GWO) based routing and clustering scheme

The paper is organized as follows. Section 2 discusses literature review. Section 3 describes the proposed Multi-Objective SMO algorithm for routing and clustering and in Section 4 the simulation results are presented. Finally, the work is summarized in Section 5

\section{Literature Review}

The energy-concerned issues of WSNs for maintaining the energy of sensor nodes and for achieving an extensive network time becomes a great challenge for the researchers. Many researchers have developed several clustering and routing approaches for enhancing the network lifetime. There are various optimization techniques proposed by the researchers to address the issues in energy consumption and network lifetime [16]. Reeta Bhardwaj et al [12] and Lipare et al presented [15] a Multi-objective algorithms. Xiaoqiang Zhao et al [27] and Lipare et al [15] proposed the Grey Wolf Optimization (GWO) and Rathore et al proposed a hybrid whale and grey wolf optimization (WGWO) [26]. Anand et al developed a genetic algorithm (GA)-based clustering and PSO based routing procedure [21]. Khoshraftar, K et al used bee colony algorithm [17] and Bhowmik, T et al used PSO to improve the performance of WSN [20]. Kim, S proposed a clustering and routing algorithms to increase the energy efficiency for achieving QoS. The main contribution if these algorithms are its scalability and responsiveness [19]. FaouziHidowssi et al (2017)[13] proposed PEAL (Power Efficient and Adaptive Latency) wireless networks, to extend network life time and achieved $47 \%$ more life time than the LEACH (Low Energy Adaptive Clustering Hierarchy) model but with more delay. Kaushik et al proposed a distance-based energy efficient and load balancing method for addressing the problems associated with compactly populated network such as interference, many transmission routes, to reduce the burden of distant nodes involved in communication for saving energy [22]. Reeta Bhardwaj et al [12] presented a Multi-objective fragmentary molecule lion calculation (MOFPL) to find a suitable team leader in various WSN locations. The proposed MOFPL considered 5, 8, 10 nodes active during WSN 2000 pressures of 50, 75, and 100 nodes, each and achieved system stability of 0.05877 and $0.06022 \mathrm{WSN}$ at 50 and 100 nodes. Seyed NaserHashemi et al [14] proposed an algorithm which used the base of the visible lattice structure of the system to solve the average rating problem. Authors reduced and balanced power 
consumption by finding the appropriate routes between each group head and sink. But their algorithm produced higher delay than other similar algorithms. Lipare et al [15] proposed the Grey Wolf Optimization (GWO) method to consolidate power and lead WSN. They used steering function statistics of specifications, current asset calculations and multicellular bias to decrease the number of bounces within the range. Lipare et al proposed Grey Wolf Optimization (GWO) approach for energy-efficient clustering and routing in WSN[15]. Authors formulated routing fitness function to minimize both the total distance traversal and the hop count and clustering fitness function to allocate the total load based on the distance between gateways and the BS. The proposed GWO-based approach is resulted with higher values of both clustering and routing. Xiaoqiang Zhao et al developed a routing algorithm based on GWO that chooses the suitable initial clusters by using various fitness functions for the nodes having varied energy [27]. The fitness values of the nodes are used as initial weights and then the weights are updated. The weight up-dation is carried out by considering both the distance between the wolves and their prey and coefficient vectors. It helped to increase the optimization capability of GWO and to guarantee the optimal choice of the cluster heads (CHs). Rathore et al proposed a hybrid whale and grey wolf optimization (WGWO)-based clustering mechanism for energy harvesting wireless sensor networks (EH-WSNs)[26]. Khoshraftar, K et al proposed a genetic algorithm to increase the clustering process of the nodes present in a wireless sensor network and moreover to find an optimal route of transmission of data through these nodes [17]. To implement this technique, the author has used the bee colony algorithm by dividing the wireless sensor network into cells of variable size. The energy consumption and compression are then evaluated in these cells and are investigated in normal mode. Based on these investigations the energy consumption is then optimized. Kavitha, A et al proposed a clustering scheme which uses the Canada geese migration principle to route data in a wireless sensor network. In this approach, the networks fields are divided into zones and based on the density of each zone, several cluster heads are selected. Those cluster heads having lower residual energy is rotated with cluster heads having high energy. This ensures that the energy consumption is uniform, and the lifetime of the nodes can be extended as all the nodes bear the same energy in themselves [18]. Sundar Rajina et al [11] suggested that network operations be managed using a load saver system with a opportunistic energy-efficient routing with load balancing (OE2-LB) that eliminates data collection delays and avoids data collection. They used smart wearable points to move loops 
efficiently. Anand et al developed a genetic algorithm(GA)-based clustering and PSO based routing procedure for improving the network lifetime. GA algorithm used distance and energy parameters to select the best cluster head $(\mathrm{CH})$. PSO based routing algorithm is used to select optimal routing paths for all relay nodes to carry communication load between $\mathrm{CH}$ and $\mathrm{BS}$ for increasing energy efficiency[21]. Bhowmik, $\mathrm{T}$ et al proposed to balance the energy present in the clustering nodes and to route the data from the cluster head to the sink. The clustering and routing in a wireless sensor network is done using the proposed improved particle swarm optimization gravitation search algorithm. Through this the energy is distributed equally throughout the entire network and optimal route to pass the data packets between the cluster head and the sink is decided using the routing algorithm. This in turn increases the network lifespan and the convergence rate [20].

From Literature, the network lifetime is found to be limited due to the limited power of sensor nodes. It is also inferred that the power gap problem may arise due to energy exhaustion during transmission. Many researchers focused on optimizing either routing or clustering through various optimization techniques such as. Particle Swarm Optimization (PSO), Grey Wolf Optimization (GWO) and Spider Monkey Optimization (SMO). Lipare et al proposed Grey Wolf Optimization (GWO) approach for energy-efficient routing and clustering in WSN [15]. In this paper, an attempt is made to optimize both the routing and clustering to balance the load and to improve the network lifetime spider monkey optimization based on the following contributions:

- Multi-objective based spider monkey optimization (MOSMO) has been proposed which uses both the routing fitness function and the clustering fitness function.

- The routing fitness function is found by incorporating both the minimum distance traversed by the gateways and minimum number of the gateway hops.

- The clustering fitness function is the minimum fitness function of gateways. The fitness function of each gateway is computed by considering both the mean load of gateways as well as the distance between gateways and BS.

\section{Multi-Objective Spider Monkey Optimization}

In this work, Multi - Objective Spider Monkey Optimization (MOSMO) has been proposed for an energy efficient clustering and routing. The objective of the proposed MOSMO is to reduce 
the total energy consumption in the network and to improve the network life time. In the proposed multi-objective-based spider monkey optimization (MOSMO) algorithm, to attain these features two fitness functions are incorporated such as Fit Routing and Fit clustering. For enhancing the energy efficiency and network lifetime, optimal load managing and routing are to be incorporated in WSN. For energy efficient routing, an optimal path needs to be selected between gateways and base station (BS). The transmission through the optimal path helps to reduce the overall energy consumption in the network. In order to reduce the consumption of total energy, an optimal path between gateways and BS is found by using Fit Routing function. In order to improve the network lifetime, load balancing, gateways near the BS are assigned a relatively smaller number of sensor nodes and gateways outside BS are assigned larger number of sensor nodes from the BS through Fit clustering function of MOSMO algorithm.

Figure 1 shows the sample model of WSN with four clusters and one BS. Each cluster includes a gateway $(\mathrm{G})$ and cluster members. As shown in the figure, these cluster members are connected to the corresponding $\mathrm{G}$ in each cluster. Gateways G1, G2, G3 and G4 correspond to 4 different clusters. Six sensor nodes are connected to G1, while three sensor nodes are present in both G2 and G3 and G4 has four sensor nodes as shown in Figure 1. Since, load of the G1 gateway is more than G2, G3 and G4. The energy consumed by G1 is greater than G2,G3 and G4. Higher energy consumption shortens the life of the G1. This affects the lifetime network. The load balancing of gateways in WSN is shown pictorially in Figure 2. If G1 fails, G2 share the load of G1 as shown in the figure 2. It can also cause premature death of G2, G3 and G4. An energy efficient approach for clustering and optimal routing becomes essential to prevent such problems with the WSN. 

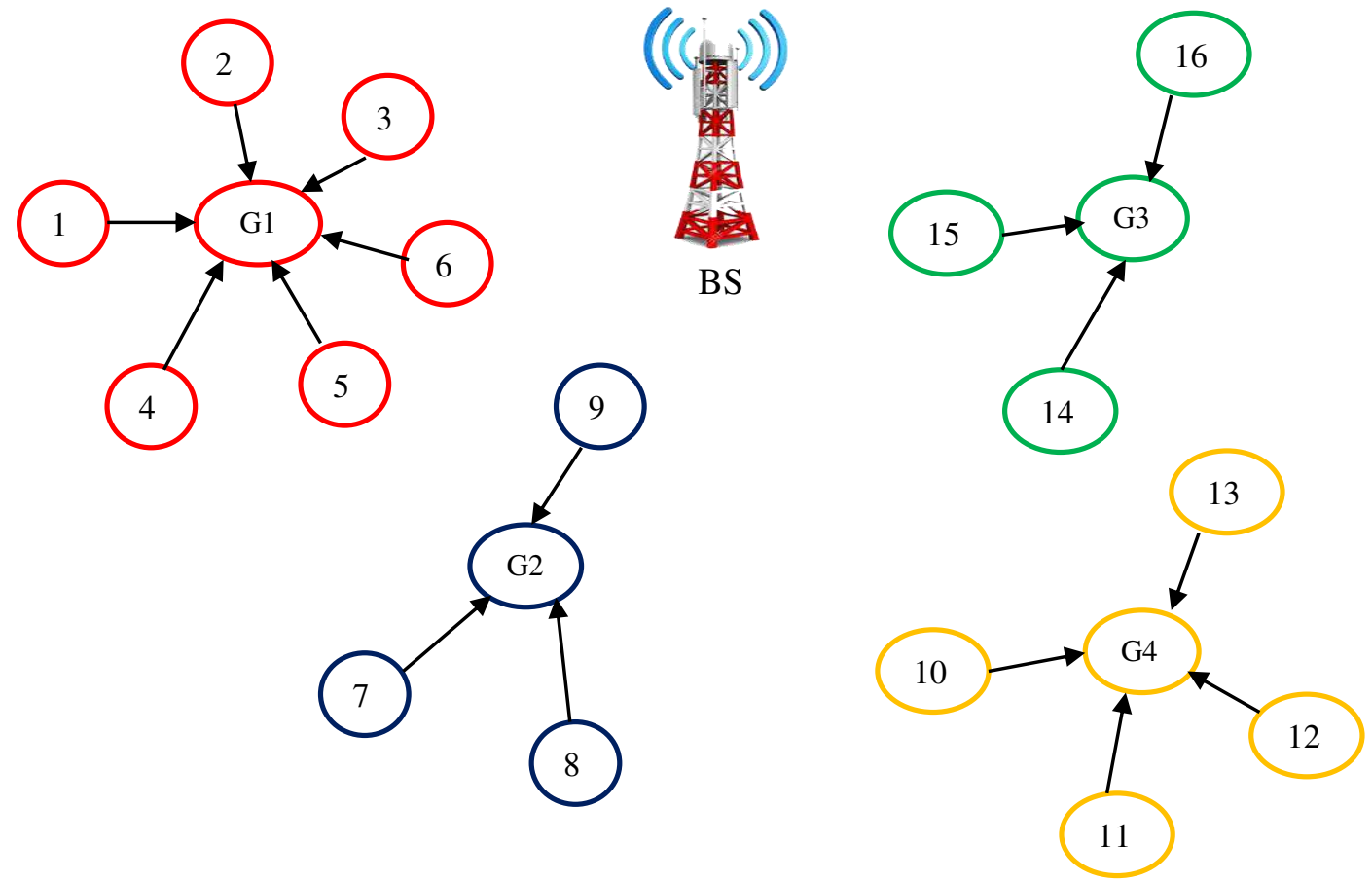

Figure 1: Sample model of WSN

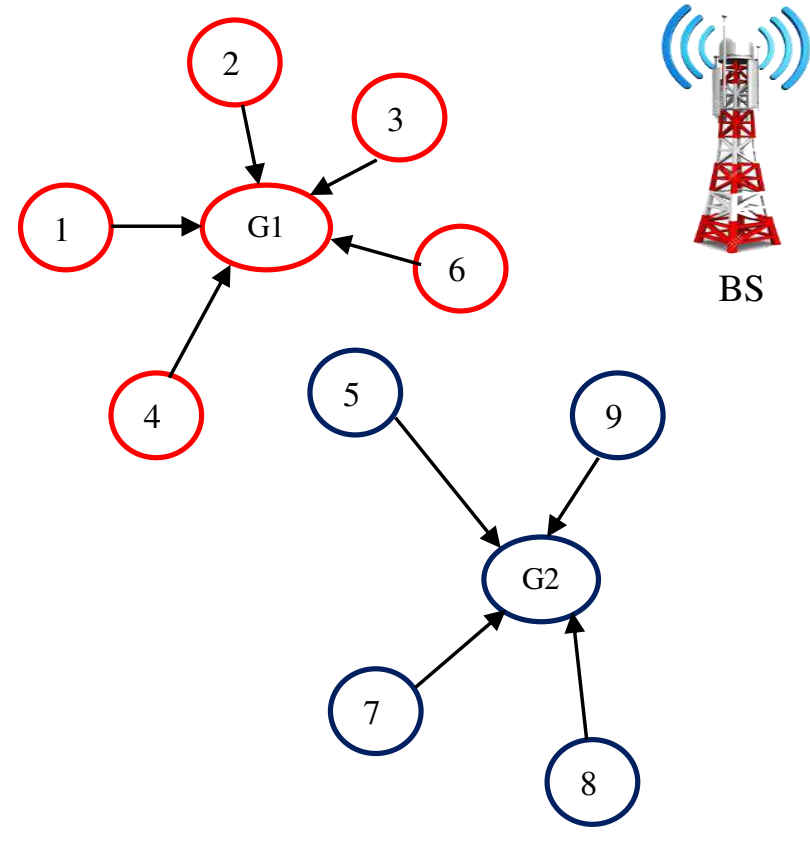

Figure 2: Load balancing in WSN

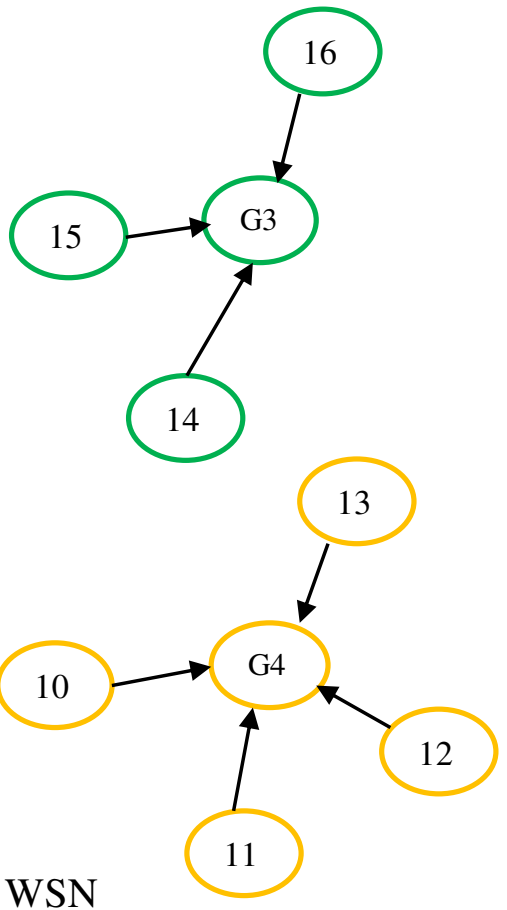




\subsection{Energy Efficient Routing using MOSMO algorithm}

The SMO algorithm incorporates the searching strategy for food for spider monkeys for optimal integration and load balancing. These searching strategies can be categorized into four stages according to the social fission-fusion social structure (FFSS) of spider monkeys. In the first step, the group checks the food classification and begins eating the food. In the second step, the positions of food collection and distribution are reviewed. In the third step, the local pioneer restores its good standing in the group. Everyone in the group began to review the diet due to the lack of renewal of the excellent position by the group pioneer. In the fourth step, the international pioneer reopens its good position. This group was divided into smaller groups because of the position (no increase in overseas pioneers in the period described earlier). The local pioneer limit (LPL) has been linked to the reduction of the problem-solving event by turning the circle into a harvest theme. On the other hand, the meeting is divided into smaller groups if the international pioneer is able to review the global pioneer limit (GPL). Negative reviews are received from the LPL and GPL of local and international pioneers to make their own decisions.

Initialization: The position $(\mathrm{P})$ of each solution or spider monkey is represented in the form of a map from one 'A' to another or BS. This solution provides access to the operating system from each gateway through the following network gateways. Each gateway starts with a random number $\left(\mathrm{U}_{\mathrm{i}, \mathrm{d}}\right)=\operatorname{rand}(0,1)$ where $1 \leq \mathrm{i} \leq \mathrm{Ms}, 1 \leq \mathrm{d} \leq \mathrm{T}$. $\mathrm{M}_{\mathrm{s}}$ is the number of major solutions. Gateway numbers are the solution to component $d$. Set the $A_{k}$ gateway to the BS route as the next gateway to $A_{d}$, indicating that $A_{d}$ is sending data to $A_{k}$. The routing path mapping is given as in equation (1)

$$
A_{k}=F\left(\operatorname{SetNextg}\left(A_{k}\right), l\right)
$$

Where $F\left(\operatorname{SetNextg}\left(A_{k}\right), l\right)$ is the face work by decreasing face of $l^{\text {th }}$ gateway from SetNextg and $l=\operatorname{Ceil}\left(U_{i, d} \times\left|\operatorname{SetNextg}\left(A_{k}\right)\right|\right)$

Fitness calculation: After the initialization of solutions, fitness value is calculated for each solution. The fitness of routing (Fit Routing) is specified to generate efficient routes from each gateway to the BS as given in equation (2). Routing path is selected by minimum distance and minimum number of hops. 


$$
\text { Fit }_{\text {Routing }}=\operatorname{Max}\left(\frac{B_{1}}{D_{\text {Sum }} * Y_{1}+A_{\text {Hop }} * Y_{2}}\right)
$$

Where, $\left(\mathrm{Y}_{1}, \mathrm{Y}_{2}\right) \in[0,1]$ such that, $\mathrm{Y}_{1}+\mathrm{Y}_{2}=1$ and $\mathrm{B}_{1}$ is a proportionality constant. Total distance traversed by gateways ( $\mathrm{D}_{\text {Sum }}$ ) is defined as in equation (3).

$$
D_{\text {Sum }}=\sum_{i=1}^{n} D\left(A_{i}, \operatorname{Nextg}\left(A_{i}\right)\right)
$$

Besides, $A_{\text {Hop }}$ taking the total number of the gateway hops of the networks

$$
A_{\text {Hop }}=\sum_{i=1}^{n} \operatorname{Nextg} \operatorname{Count}\left(A_{i}\right)
$$

According to equation (2), the solution with maximum fitness function is considered as the optimal solution or optimal routing path.

Update the solution: This renewal phase includes the components such as (i) to (vi) (Local pioneer phase, Global pioneer phase, Learning phase of global pioneer, Learning phase of local pioneer, Decision phase of local pioneer, Decision phase of global pioneer) of this place. The components of this algorithm are described below.

(i) Local pioneer phase: At this point, each spider or monkey determines its new position based on the experience of local pioneers and local team members as given in equation (5).

$$
P_{\text {new }}=\left\{\begin{array}{lr}
\boldsymbol{P}_{i, d, d}+R_{1} \times\left(L P_{k, d}-P_{i, d}\right)+R_{2} \times\left(P_{j, d}-P_{i, d}\right) & \text { if } R_{1} \geq P R \\
P_{i, d} & \text { otherwise }
\end{array}\right.
$$

where, $\mathrm{R}_{1}$ and $\mathrm{R}_{2}$ represent the random numbers $[0,1]$ and $[-1,1], \mathrm{LP}_{\mathrm{k}}, \mathrm{d}$ represents the position of the local pioneer in $\mathrm{k}^{\text {th }}$ magnitude. $\mathrm{d}^{\text {th }}$ represents the spider monkey randomly selected in group $\mathrm{P}_{\mathrm{j}}, \mathrm{d}$ on the $\mathrm{j}^{\text {th }}$ scale $\mathrm{d}^{\text {th }}$. PR represents a measure of certain factors that indicate the amount of perturbation. 
(ii) Global pioneer phase: The local pioneer class is followed by this taking, known as the world pioneer class on the point, all the spider monkeys update their positions based on the experiences of local team members and international leaders as in equation (6).

$$
P_{n e w_{i, d}}=P_{i, d}+R_{1} \times\left(G P_{d}-P_{i, d}\right)+R_{2} \times\left(P_{j, d}-P_{i, d}\right)
$$

where, $G P_{d}$ represents the position of international pioneers in the $d^{\text {th }}$ dimension. Spider monkeys are revived according to the following number of possibilities. This can include job stress. The solution or the spider monkey is more likely to reuse its position. This number of opportunities $\left(\mathrm{Op}_{i}\right)$ is calculated as in equation (7).

$$
O p_{i}=\frac{F i t_{i}}{\max -F i t} \times 0.9+0.1
$$

(iii)Learning phase of global pioneer: At this stage, the position of overseas pioneers is being reviewed, and a powerful spider monkey has been selected as an international pioneer. If the levels of pioneers overseas are not reviewed, the international border value of a good local solution will be increased by 1 .

(iv)Learning phase of local pioneer: Given the role of the local leader, the local limit on the number of pilot land solutions will be increased by 1 .

(v) Decision phase of local pioneer: If the number of local leaders exceeds the number of local leaders, the status of all team members is determined at random or based on information received from local and foreign leaders as in equation (8).

$$
P_{\text {newi,d }}=P_{i, d}+R_{1} \times\left(G P_{d}-P_{i, d}\right)+R_{1} \times\left(P_{i, d}-L P_{j, d}\right)
$$

From equation (8), it is inferred that spider monkey position is updated and leading towards the global pioneer position.

(vi)Decision phase of global pioneer: At this phase, the population is divided into smaller groups where the border is larger than the international pioneer limit. A local pioneer limit is set for selecting a local pioneer from each group. If the position of the pioneers abroad is not reviewed, the international pioneer will join all the groups and form a group. 
Termination: The process is repeated until the optimal solution or routing path obtained. Then result is attained, the algorithm will be terminated.

\subsection{Energy Efficient clustering using SMO algorithm}

The SMO-based clustering algorithm expoits the solution of the proposed route algorithm. The base station measures the load on the gateways using the route solution obtained from the proposed algorithm.

Initialization: In, the Clustering algorithm, the size of the solution is equivalent to the number of sensor nodes $(\mathrm{N})$. In the first method, all sensor space is assigned a random number $\mathrm{V}_{\mathrm{i}, \mathrm{d}}=$ $\operatorname{rand}(0,1)$ where $1 \leq \mathrm{i} \leq \mathrm{M}_{\mathrm{c}}, 1 \leq \mathrm{d} \leq \mathrm{N}$. $\mathrm{M}_{\mathrm{c}}$ is the number of initial clustering solutions. Compound D contains the index of the sensor node (s) and the corresponding inputs to the gateway are in the same cluster as the cluster head (AK), indicating that $S_{d}$ sends data to the $G_{k}$. The mapping of the sensor node and gateway is given in equation (9).

$$
A_{k}=I\left(\operatorname{Coms}\left(S_{d}\right), l\right)
$$

Where $I\left(\operatorname{Coms}\left(S_{d}\right), l\right)$ is an indexing function that yields index of $\mathrm{n}^{\text {th }}$ gateway from the set of $\operatorname{Coms}\left(S_{d}\right)$ and $l=\operatorname{Ceil}\left(V_{i, d} \times \mid \operatorname{Coms}\left(S_{d}\right)\right)$. Since the size of the solution is equal to the number of sensor nodes, the introduction and disassembly of the nodes results in a difference in the solution size necessitating the need for reassembly.

Fitness calculation: After initialization, a new fitness grouping function (Fitness clustering) is evaluated for each solution as in equation (10). The closest gateways to the BS are connected to the fewest sensor nodes, while the remote gateways are connected to the most sensor nodes. Gateway outside BS sends data from gateway outside BS. Thus the gateway minimizes its own load as it is connected to a low sensor node.

$$
\text { Fit }_{\text {Clustering }}=\operatorname{Min}\left(\frac{\sum_{i=1}^{n} F_{i t_{\text {Clustering }}\left(A_{i}\right)}}{n}\right)
$$


Where, Fit Clustering $_{(}\left(A_{i}\right)$ represents the clustering fitness function for the gateway and is calculated as in equation (11).

$$
\text { Fit }_{\text {Clustering }}\left(A_{i}\right)=H_{2} \times\left|L\left(A_{i}\right)-D\left(B S, A_{i}\right) * \mu\right|
$$

Where, $\mathrm{H}_{2}$ denotes the proportionality constant, $\mu$ represents the mean load over all gateways according to the distance between gateways and BS. This mean load $(\mu)$ is calculated as in equation (12).

$$
\mu=\frac{\sum_{i=1}^{n} L\left(A_{i}\right)}{n}
$$

Update the solution: The solution is updated continually as in routing phase using equations (5) through (8) until the optimal solution is attained.

Termination: Once an optimal solution or clustering is found. The algorithm will be terminate.

\section{Results and Discussions}

The proposed Multi-Objective Spider Monkey Optimization (MOSMO) is being implemented on the MATLAB platform. Algorithm is set to run in simulation environment as given Table 1. Each sensor node can transmit the power up to the distance of 250 meters. Each sensor node is equipped with a light-emitting that produces the same radio frequency energy in all directions.

Table 1: Simulation Environment

\begin{tabular}{|c|c|}
\hline Parameter & Values \\
\hline Area Size & $1000 \mathrm{~m} \times 1000 \mathrm{~m}$ \\
\hline Routing Protocol & AODV \\
\hline MAC & $802 \_11$ \\
\hline Antenna & Omni Antenna \\
\hline Radio Propagation Model & Two Ray Ground \\
\hline Packet size & 512 bytes \\
\hline Initial transmitting power & $0.660 \mathrm{~W}$ \\
\hline
\end{tabular}




\subsection{Performance analysis}

\begin{tabular}{|c|c|}
\hline Initial receiving power & $0.395 \mathrm{~W}$ \\
\hline Initial energy & $10.3 \mathrm{~J}$ \\
\hline
\end{tabular}

The performance of proposed MOSMO in routing and clustering scheme is evaluated in terms delay, power consumption, delivery ratio, and throughput and network lifetime with existing Particle Swarm Optimization (PSO) and Grey Wolf Optimization (GWO) algorithms under various node densities. Fig. 3 shows delay analysis with various density nodes. It is inferred from Fig. 3 that the delay increases as the number of sensor nodes increases. However, the delay of proposed MOSMO-based algorithm is $18 \%$ and $27 \%$ lower than GWO and PSO respectively. As the MOSMO algorithm optimally clusters the nodes using Fit Clustering function and routes the packets efficiently using Fit routing function. It achieves good convergence speed and lesser computational complexity than the GWO and PSO. Due to the efficient load balancing with the efficient clustering algorithm, transmission delay of the data packet is reduced.

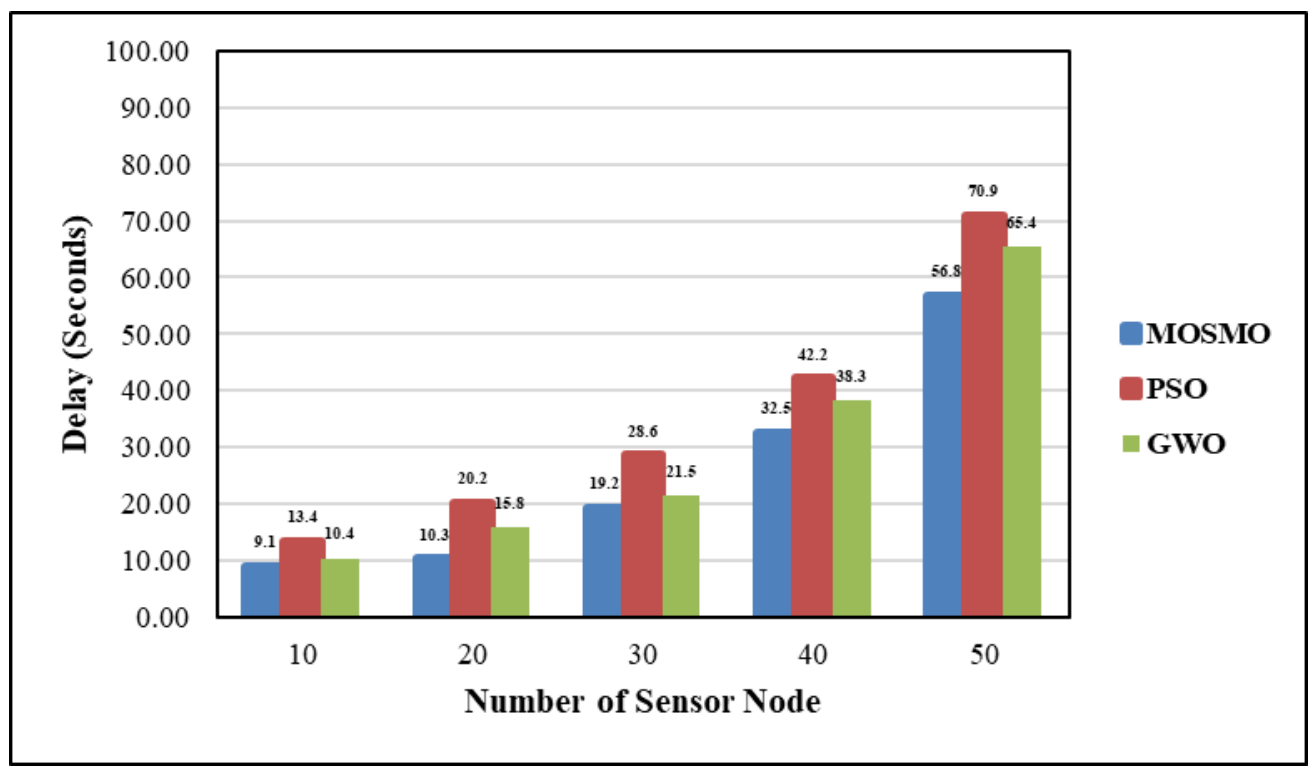

Figure 3 Delay incurred with respect to Node density 


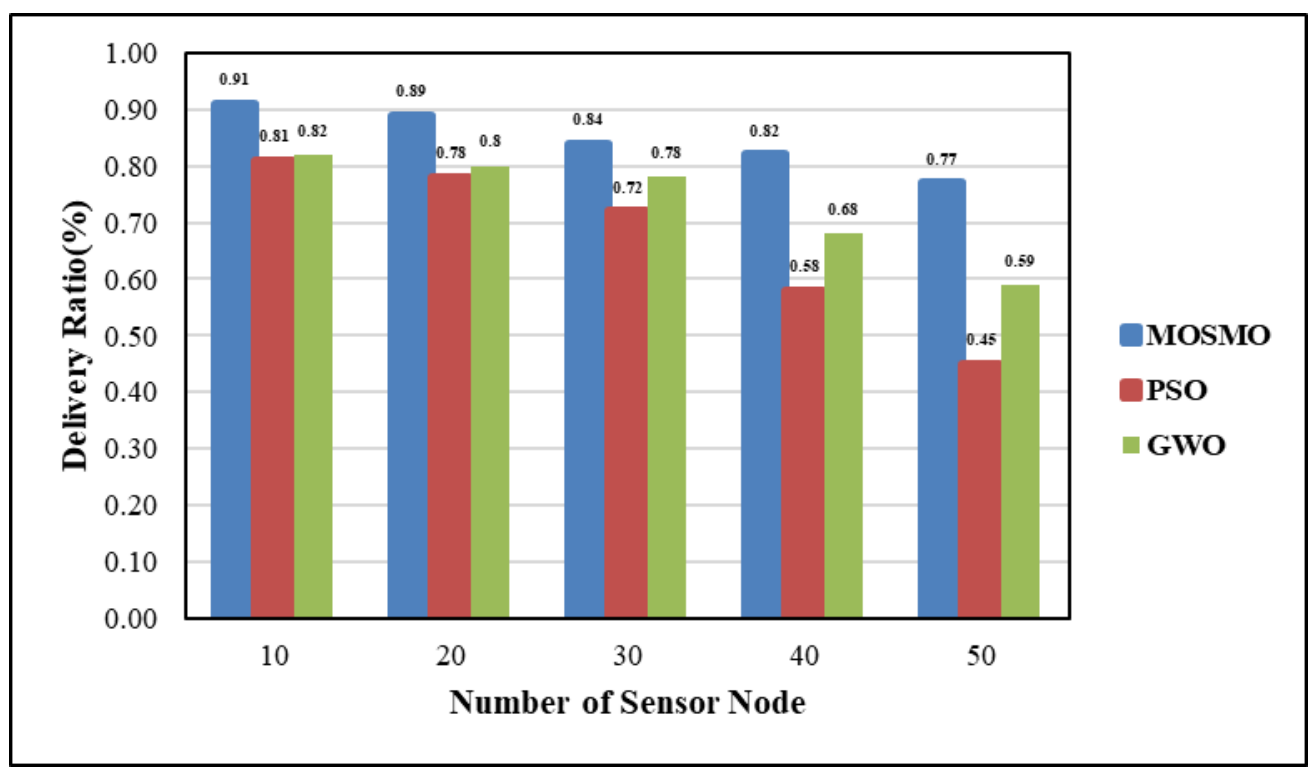

Figure 4 Impact of Node Density on Delivery Ratio

The analysis of delivery ratio of the proposed MOSMO and that of the existing PSO and GWO based on the density of the sensor nodes is shown in Figure 4. The optimal route selection using the proposed SMO resulted in an $15 \%$ and $26 \%$ increase in the delivery rate compared to the existing GWO and PSO respectively.

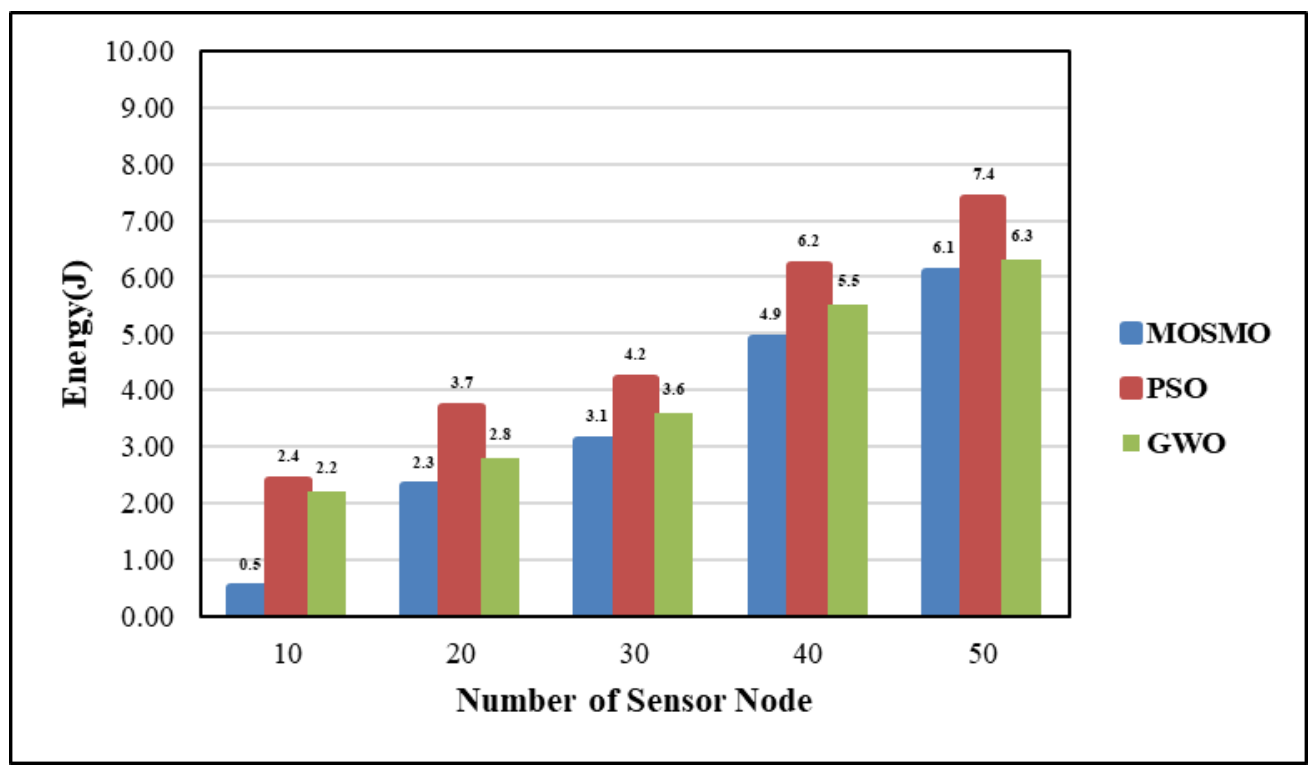

Figure 5 Impact of Node Density on Energy Consumption 
Figure 5 shows a comparison of the energy consumption of the clustering and routing scheme with different algorithms. The energy consumption of the proposed MOSMO has been reduced by $17 \%$ and $29 \%$ respectively as compared to GWO and PSO due to the proposed clustering scheme and the choice of better route using load balancing at the entry point of network.

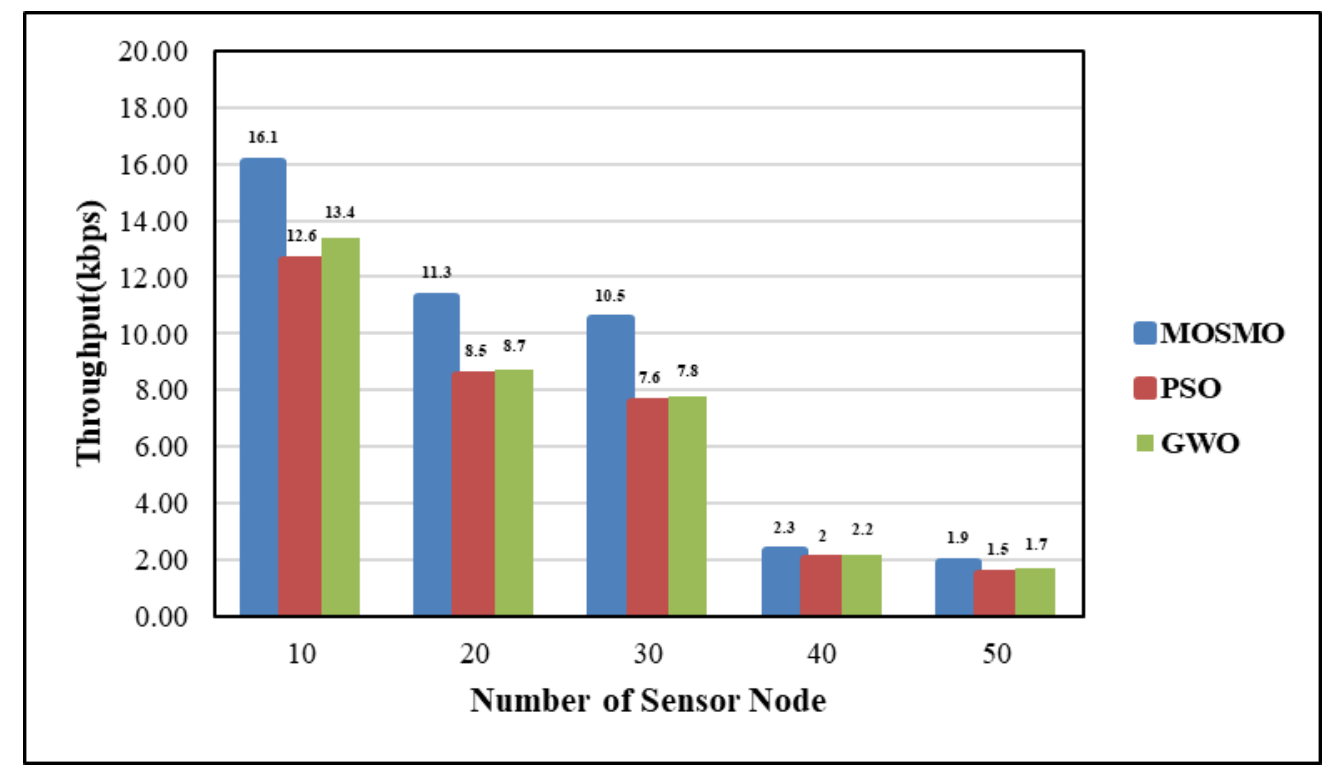

\section{Figure 6 Throughput achieved with respect to Node Density}

Throughput analysis with various density of nodes is shown in the Figure 6. Throughput is decreased when the number of sensor nodes increases. The throughput of the proposed MOSMO based clustering and routing scheme is increased to $24 \%$ and $30 \%$ compare to existing GWO and PSO based clustering and routing scheme respectively. The reason for this achievement of throughput improvement are convergence speed, selection of optimal routing path between source and BS. 


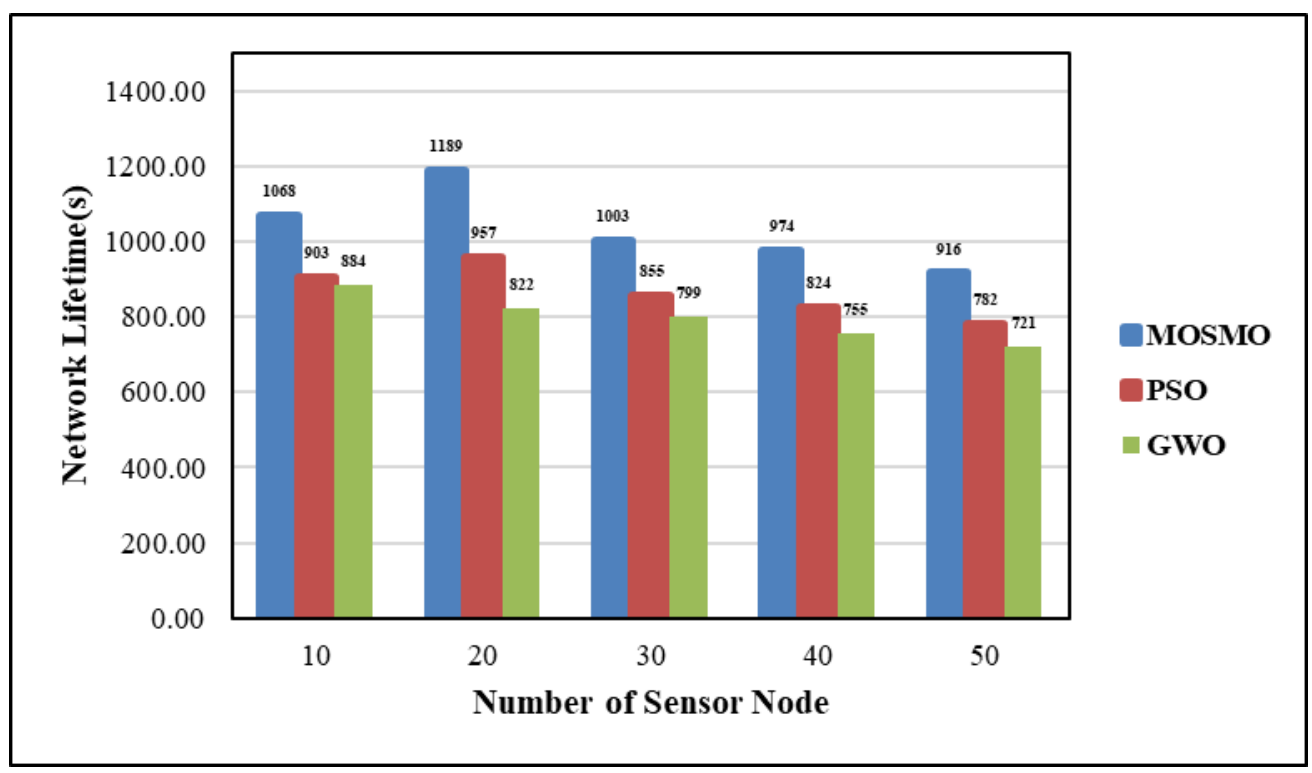

Figure 7 Impact of Node Density on Network Lifetime

The variation in life time of the network with various density of nodes is shown in Figure 7 The network lifetime of the proposed SMO based clustering and routing scheme is increased to $29 \%$ and $19 \%$ than that of existing GWO and PSO based clustering and routing schemes respectively. It is due to the load balancing among the gateways.

\section{Conclusions and Future work}

In this paper, the major issues of WSN such as heavy traffic load around the gateways and network lifetime are addressed using multi-objective spider monkey optimization (MOSMO) algorithm based routing and clustering scheme. Using the MOSMO based routing, the optimal route between the source and the BS is established to increase the energy efficiency and lifetime of the network. In addition, clustering the sensor nodes are assigned to the gateway near the BS and a large number of sensor nodes are assigned to the gateway away from the BS. The routing fitness function is found by incorporating both the minimum distance traversed by the gateways and minimum number of the gateway hops. The clustering fitness function is the minimum fitness function of gateways. The fitness function of each gateway is computed based on both the mean load of gateways as well as the distance between gateways and BS. The proposed SMO based routing and clustering scheme outperformed the existing PSO and GWO based routing and clustering schemes in terms of delay, energy consumption, delivery ratio, throughput and 
network lifetime. The results proved that, the reduction in delay and energy consumption is about $18 \%$ and $17 \%$ respectively whereas improvement in delivery ratio, throughput and network life time is about $15 \%, 24 \%$ and $19 \%$ respectively when compared to the existing PSO and GWO methods. The proposed MOSMO algorithm is useful for evolving technologies with larger network size of IoT (Internet of Things) to optimize consumption of energy by sensor nodes. The work can be further extended in developing GA-MOSMO based algorithm to determine the optimum routing path and to improve the network lifetime and for QoS provisioning.

\section{Declarations:}

Funding (information that explains whether and by whom the research was supported) - Not Applicable Conflicts of interest/Competing interests (include appropriate disclosures) - Not Applicable Availability of data and material (data transparency) - Generated by NS-2 Simulator Code availability - Custom Code

Authors' contributions: Conceptualization, A.R., D.S.; Methodology, A.R., D.S.; Coding, A.R., D.S; Result analysis, A.R., D.S.; Manuscript preparation, A.R., D.S;

\section{References}

1. Raghavendra, Cauligi S., Krishna M. Sivalingam, and TaiebZnati, eds. Wireless sensor networks. Springer, 2006.

2. Akyildiz, Ian F., et al. "Wireless sensor networks: a survey." Computer networks 38.4 (2002): 393-422.

3. Merrett, Geoff V., et al. "Resource aware sensor nodes in wireless sensor networks." Journal of Physics: Conference Series. Vol. 15. No. 1. IOP Publishing, 2005.

4. Tang, Jian, Bin Hao, and Arunabha Sen. "Relay node placement in large scale wireless sensor networks." Computer communications 29.4 (2006): 490-501.

5. Kuila, Pratyay, Suneet K. Gupta, and Prasanta K. Jana. "A novel evolutionary approach for load balanced clustering problem for wireless sensor networks." Swarm and Evolutionary Computation 12 (2013): 48-56. 
6. Kuila, Pratyay, and Prasanta K. Jana. "Energy efficient clustering and routing algorithms for wireless sensor networks: Particle swarm optimization approach." Engineering Applications of Artificial Intelligence 33 (2014): 127-140.

7. Edla, Damodar Reddy, et al. "An efficient load balancing of gateways using improved shuffled frog leaping algorithm and novel fitness function for WSNs." IEEE Sensors Journal 17.20 (2017): 6724-6733.

8. Edla, Damodar Reddy, AmrutaLipare, and RamalingaswamyCheruku. "Shuffled complex evolution approach for load balancing of gateways in wireless sensor networks." Wireless Personal Communications 98.4 (2018): 3455-3476.

9. Mohemed, Reem E., et al. "Energy-efficient routing protocols for solving energy hole problem in wireless sensor networks." Computer Networks 114 (2017): 51-66.

10. Chen, Guihai, et al. "An unequal cluster-based routing protocol in wireless sensor networks." Wireless Networks 15.2 (2009): 193-207.

11. Raj, A. Sundar, and M. Chinnadurai. "Energy efficient routing algorithm in wireless body area networks for smart wearable patches." Computer Communications 153 (2020): 8594.

12. Bhardwaj, Reeta, and Dinesh Kumar. "MOFPL: Multi-objective fractional particle lion algorithm for the energy aware routing in the WSN." Pervasive and Mobile Computing 58 (2019): 101029.

13. Hidoussi, Faouzi, et al. "PEAL: power efficient and adaptive latency hierarchical routing protocol for cluster-based WSN." Wireless Personal Communications 96.4 (2017): 49294945.

14. Yarinezhad, Ramin, and SeyedNaserHashemi. "Solving the load balanced clustering and routing problems in WSNs with an fpt-approximation algorithm and a grid structure." Pervasive and Mobile Computing 58 (2019): 101033.

15. Lipare, Amruta, Damodar Reddy Edla, and VenkatanareshbabuKuppili. "Energy efficient load balancing approach for avoiding energy hole problem in WSN using Grey Wolf Optimizer with novel fitness function." Applied Soft Computing 84 (2019): 105706.

16. Al Aghbari, Zaher, et al. "Routing in wireless sensor networks using optimization techniques: A survey." Wireless Personal Communications (2019): 1-28. 
17. Khoshraftar, Kobra, and BehnamHeidari. "A Hybrid Method Based on Clustering to Improve the Reliability of the Wireless Sensor Networks." Wireless Personal Communications 113.2 (2020): 1029-1049.

18. Kavitha, A., KoppalaGuravaiah, and R. LeelaVelusamy. "CS-CGMP: Clustering Scheme Using Canada Geese Migration Principle for Routing in Wireless Sensor Networks." Wireless Personal Communications 115.2 (2020): 1363-1384.

19. Kim, Sungwook. "Adaptive online sensor clustering and routing algorithms for QoS provisioning and energy efficiency." Wireless Personal Communications 63.4 (2012): 965-975.

20. Bhowmik, Tanima, and Indrajit Banerjee. "An Improved PSOGSA for Clustering and Routing in WSNs." Wireless Personal Communications (2020): 1-29.

21. Anand, Veena, and Sudhakar Pandey. "New approach of GA-PSO-based clustering and routing in wireless sensor networks." International Journal of Communication Systems 33.16 (2020): e4571.

22. Kaushik, Ajay, S. Indu, and Daya Gupta. "A grey wolf optimization approach for improving the performance of wireless sensor networks." Wireless Personal Communications 106.3 (2019): 1429-1449.

23. Sharma, Harish, GarimaHazrati, and Jagdish Chand Bansal. "Spider monkey optimization algorithm." Evolutionary and swarm intelligence algorithms. Springer, Cham, 2019. 4359.

24. Wang, Jin, et al. "An improved routing schema with special clustering using PSO algorithm for heterogeneous wireless sensor network." Sensors 19.3 (2019): 671.

25. Daneshvar, SM Mahdi H., PardisAlikhahAhariMohajer, and Sayyed Majid Mazinani. "Energy-efficient routing in WSN: A centralized cluster-based approach via grey wolf optimizer." IEEE Access 7 (2019): 170019-170031.

26. Rathore, Rajkumar Singh, et al. "Hybrid WGWO: whale grey wolf optimization-based novel energy-efficient clustering for EH-WSNs." EURASIP Journal on Wireless Communications and Networking 2020 (2020): 1-28.

27. Zhao, Xiaoqiang, et al. "Routing protocol for heterogeneous wireless sensor networks based on a modified grey wolf optimizer." Sensors 20.3 (2020): 820. 
Figures
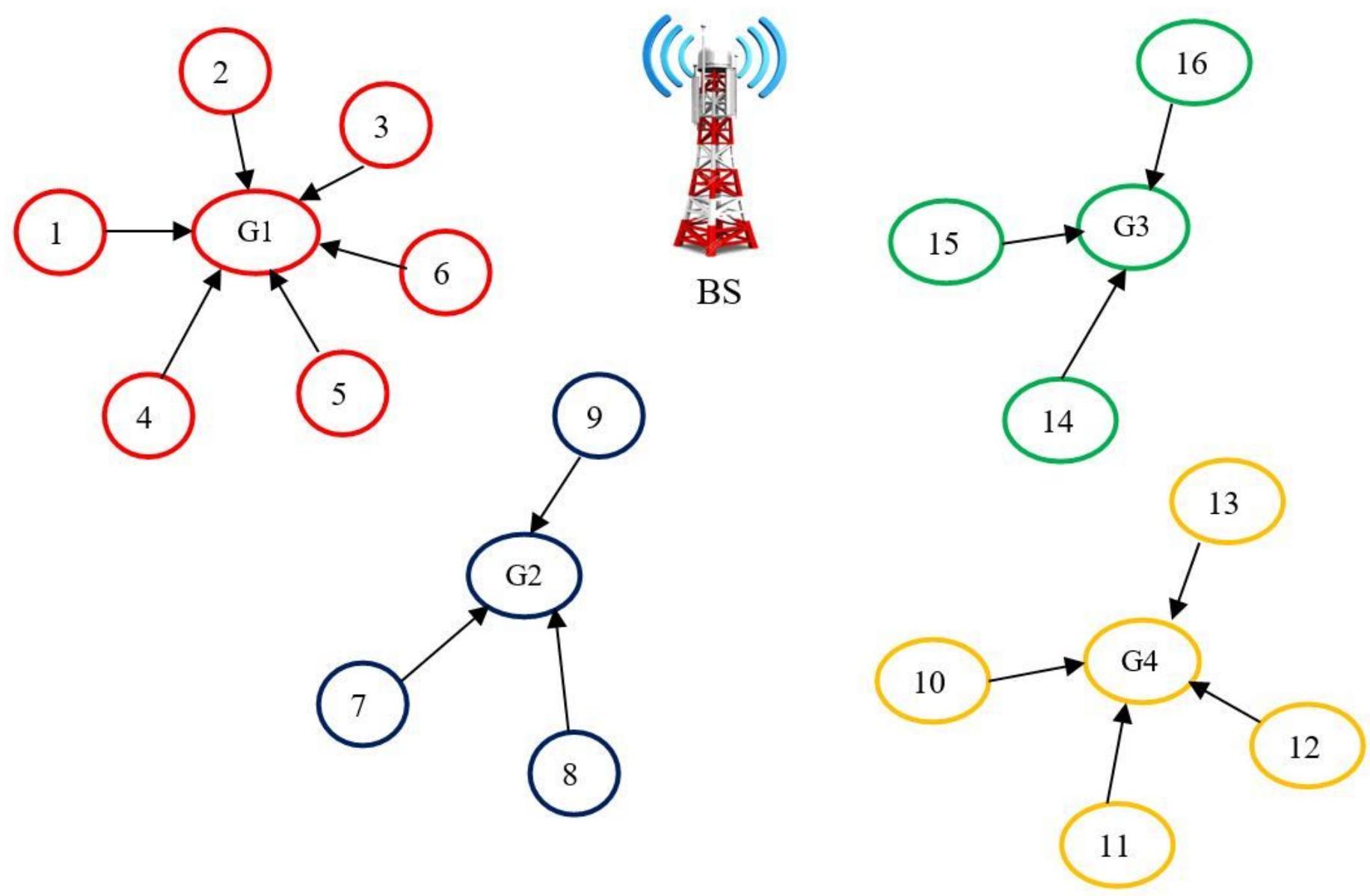

Figure 1

Sample model of WSN 

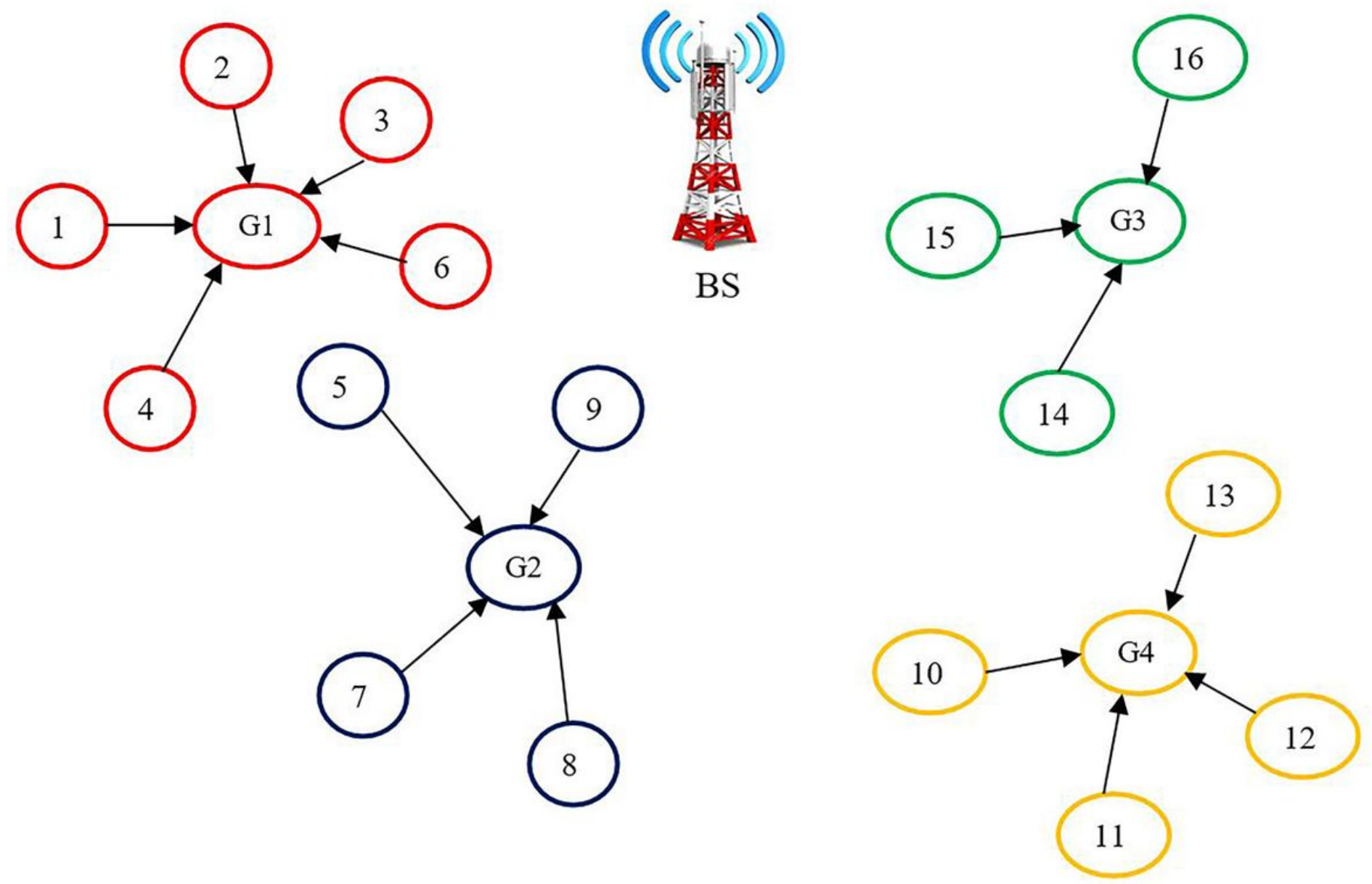

Figure 2

Load balancing in WSN 


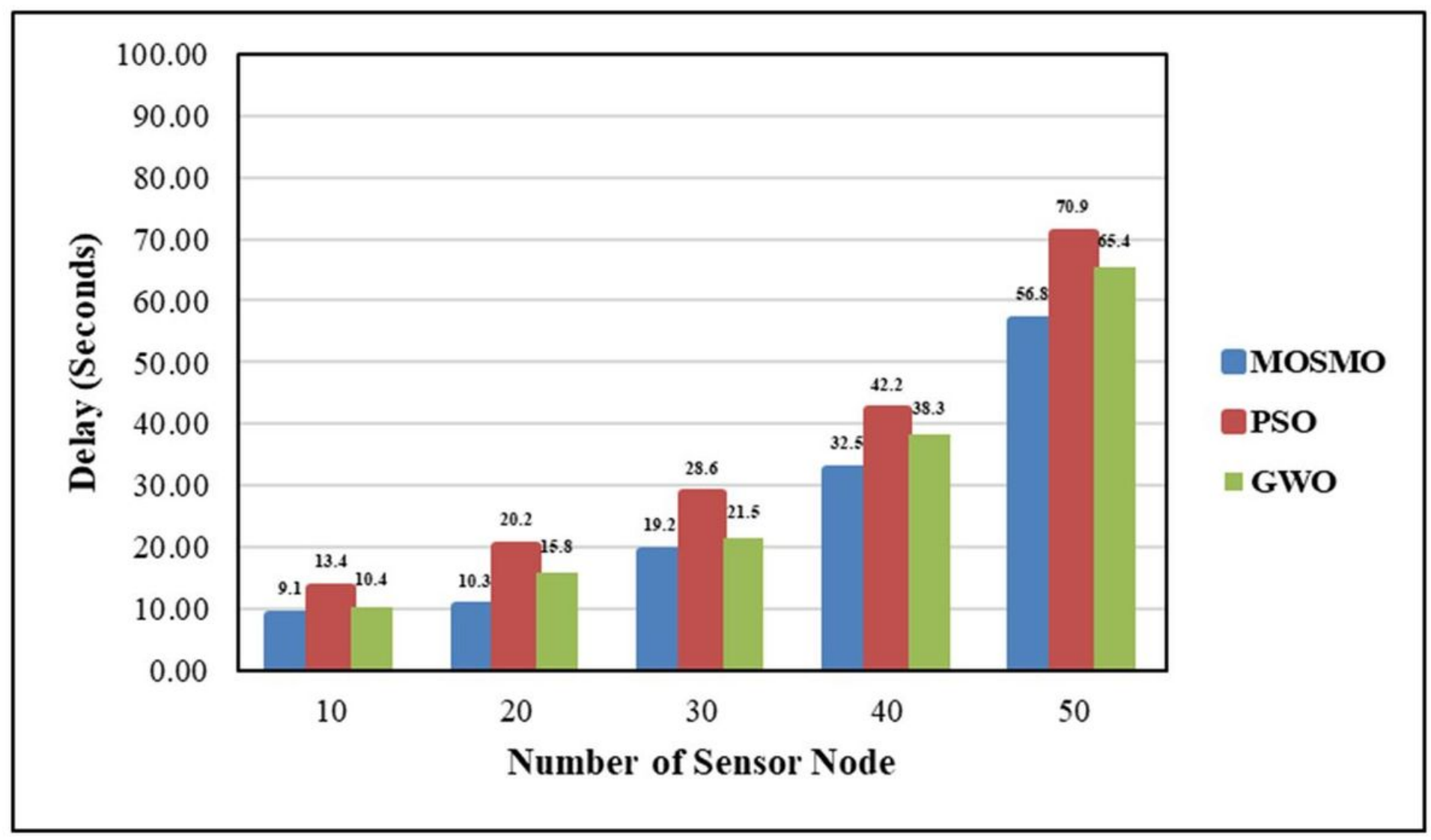

Figure 3

Delay incurred with respect to Node density 


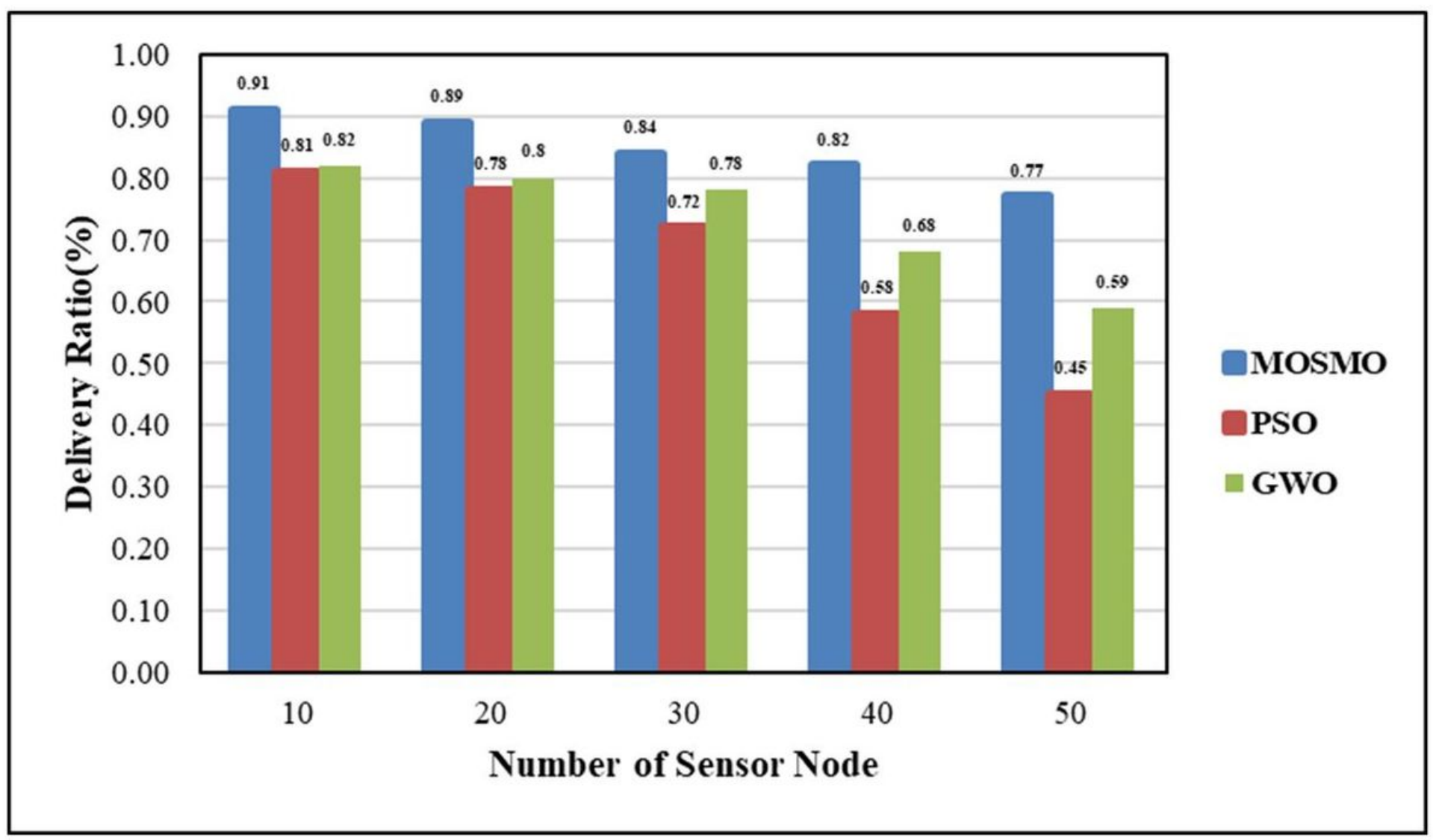

Figure 4

Impact of Node Density on Delivery Ratio 


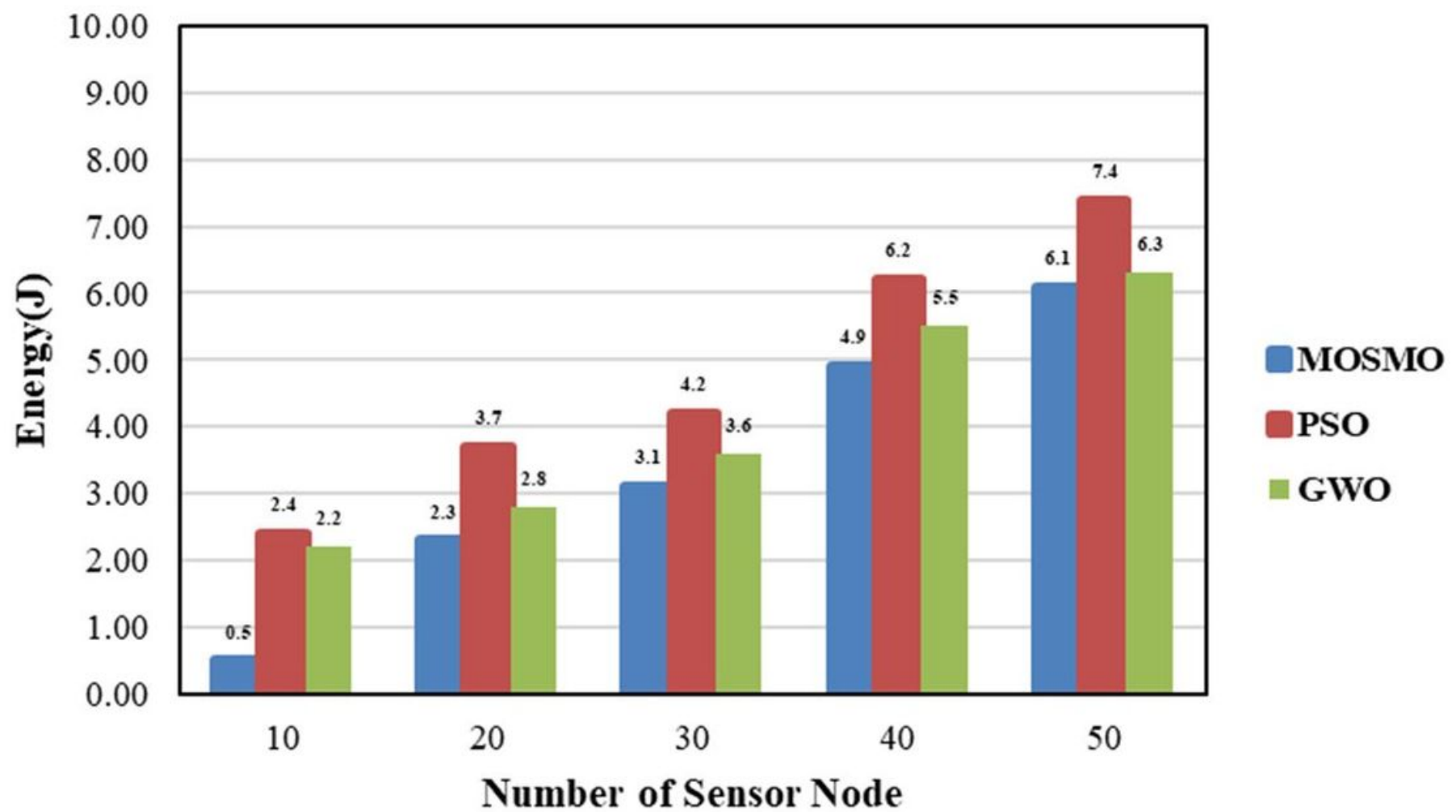

Figure 5

Impact of Node Density on Energy Consumption

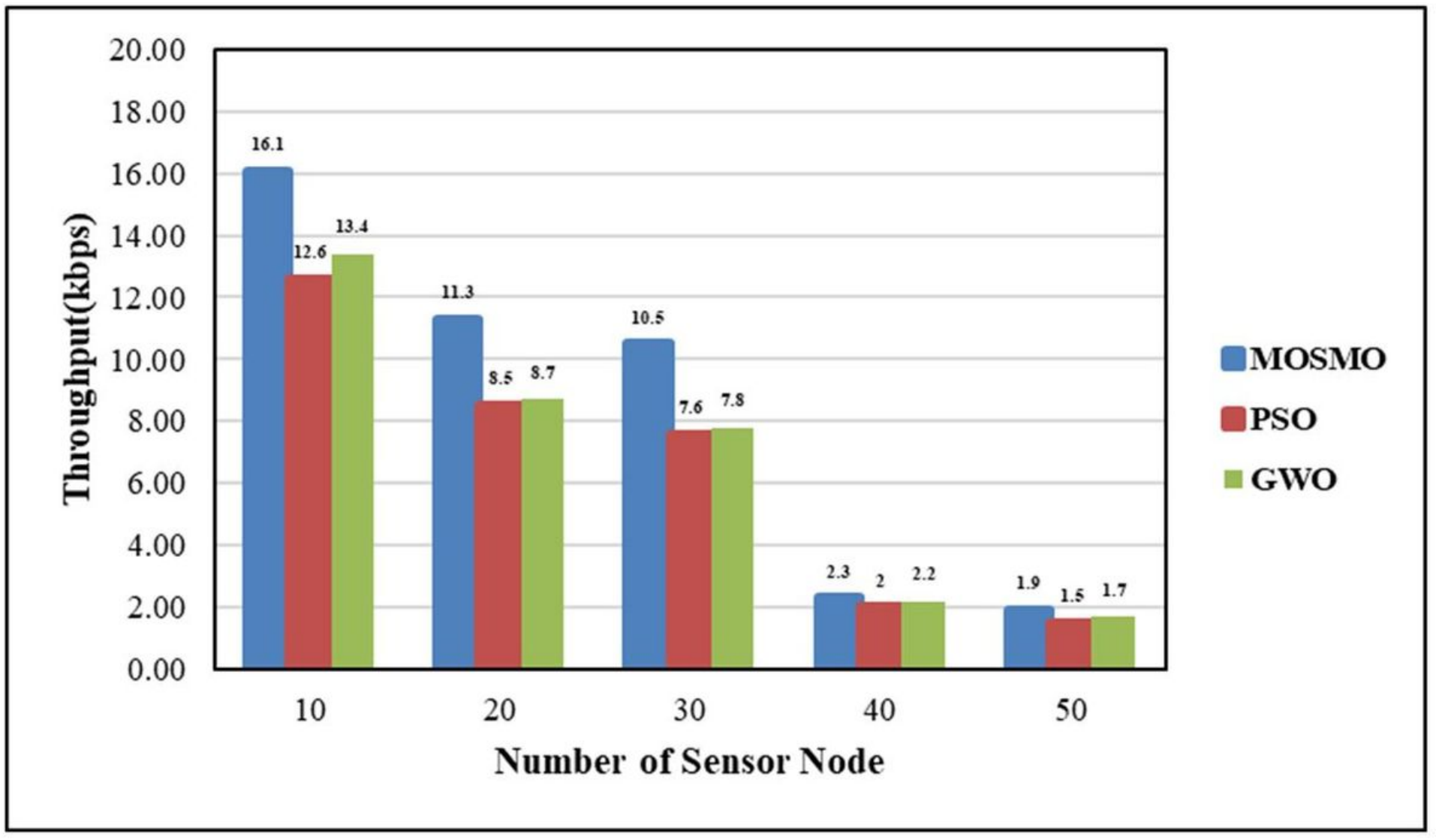


Figure 6

Throughput achieved with respect to Node Density

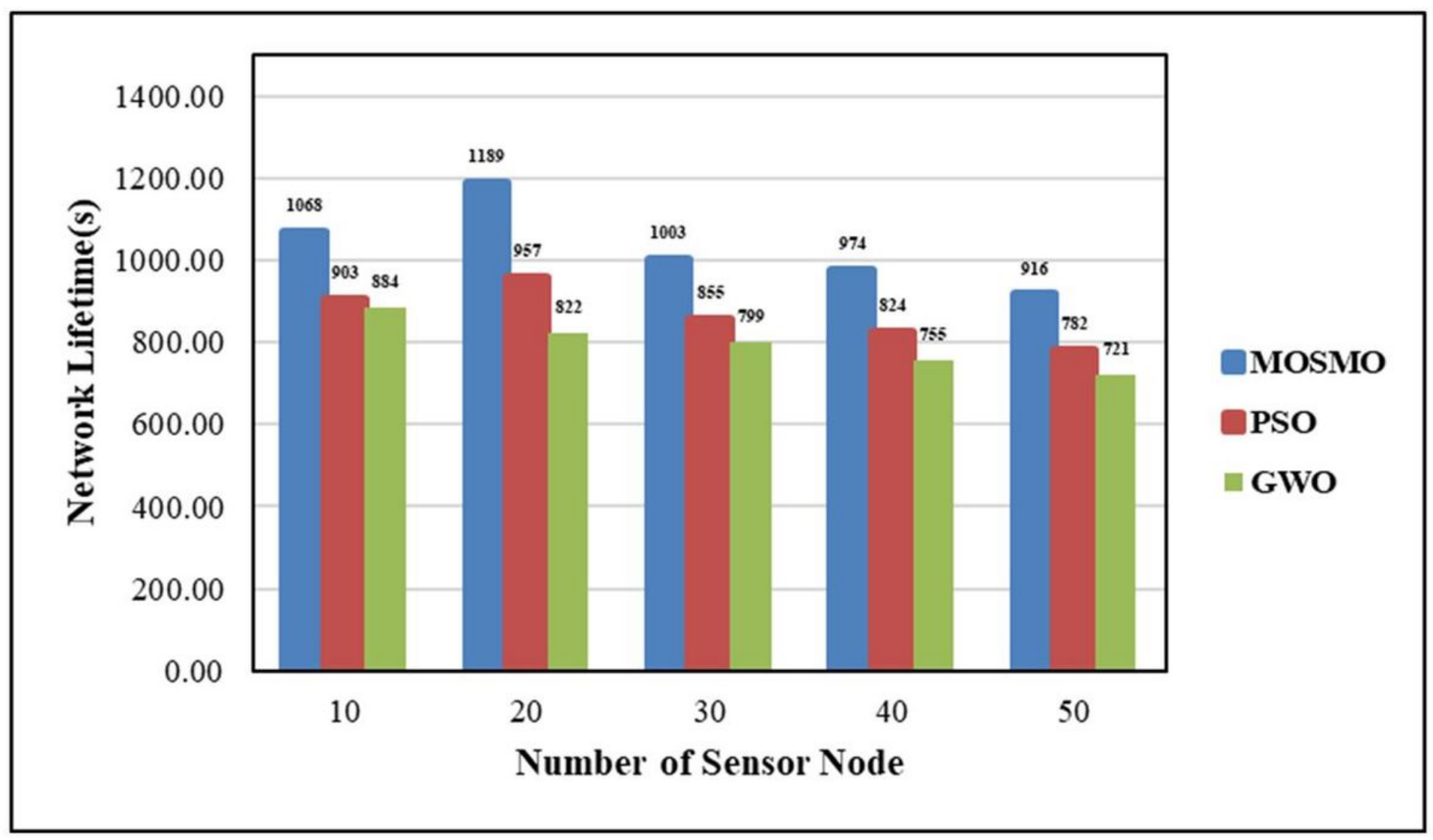

Figure 7

Impact of Node Density on Network Lifetime 